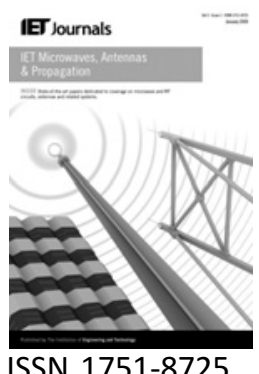

\title{
Monte Carlo simulation of an radio frequency identification system with moving transponders using the partial element equivalent circuit method
}

\author{
$\begin{array}{lll}\text { T. Lindgren } & \text { B. Kvarnström } & \text { J. Ekman } \\ & \end{array}$ \\ ${ }^{1}$ Department of Computer Science and Electrical Engineering, Luleå University of Technology \\ ${ }^{2}$ Department of Business Administration and Social Sciences, Lulea University of Technology \\ E-mail: tore.lindgren@ltu.se
}

\begin{abstract}
When designing an radio frequency identification system it is important to take both the position and the movement of the transponders into account. In this study, a simulation method that enables the description of a complete RFID system including moving and rotating transponders as well as a complex, industrial environment is presented. By using the partial element equivalent circuit method to calculate the magnetic field generated by the reader antenna and describing the transponders using a magnetic dipole, it is possible to use the Monte Carlo method to describe the dynamic behaviour of the complete system. The method is used in this study to describe the difference in performance between two different reader antennas and these results are also compared to measurements of similar systems operating in an industrial environment. The difference in performance between the two systems is similar in both the simulations and the measurements. A small discrepancy was seen between the results from the simulations and the measurements which is for the most part because of the limited read rate of the RFID systems used in the measurements.
\end{abstract}

\section{Introduction}

The electromagnetic properties of a device may be significantly altered because of movement of one or more components. This is of particular concern to the radio frequency identification (RFID)-community where the readability of the RFID transponders depends strongly on their orientation and position with respect to the reader antenna. Typically, the orientation, position and movement of the transponder is known with some statistical distribution. It is therefore desired to have a model capable of predicting the performance of a system in terms of the probability that a transponder is detected by the reader or some other statistical property of interest.

The ability to predict the performance of an RFID system in a realistic environment has gained significant attention recently. This is of particular concern for the process industry, where there is little or no control over the orientation, position and movement of the transponders. Consider for example [1] where the use of RFID systems to trace iron ore pellets in a product chain is evaluated experimentally. The results indicate that RFID systems can be used for this type of application. In [2] the readability of RFID transponders when placed on different types of goods was investigated experimentally. In that study, a $100 \%$ read rate was never achieved regardless of configuration. Similar results were obtained in [3], where measurements in an anechoic chamber were compared to measurements in a warehouse environment. Improving the reliability of RFID systems by using redundancy (i.e. multiple reader antennas) was suggested and evaluated experimentally in [4]. The performance of an RFID transponder is also affected when located close to a metallic 
surface, and this effect was quantified in [5] using finite element method simulations and measurements. A method to characterise an ultra high frequency (UHF) RFID system is proposed in [6]. This method uses a combination of free space measurements of the readability of transponders and statistical models of fading because of multipath effects for example.

Although the focus of the investigations presented in [2-6] has mainly been in the UHF band (typically $860-960 \mathrm{MHz}$ ), the problems are similar in the lowfrequency bands (125 and $134.5 \mathrm{kHz}$ ). This was assessed in [7] where a reader antenna design suitable for an animal identification system was proposed. The studies mentioned so far have relied heavily on measurements to analyse the performance of an RFID system in a realistic environment. Sources of errors in simulations of RFID systems are presented in [8]. Careful interpretation of simulation results is called for owing to the fact that simulations often need to be simplified in order to reduce the computational time.

The purpose of this paper is to present a simulation method capable of describing the performance of an RFID system, operating in an industrial environment, in statistical terms. The approach chosen is similar to the one used in [9], where the Monte Carlo method is used to assess the effects of physical damage on antennas. In the present paper, the partial element cquivalent circuit (PEEC) method (see [10-12]) is used to calculate the current in, and the magnetic field created by, the reader antenna. This method is based on the integral formulation of Maxwell's equations which enables modelling of the whole system without having to mesh the air between the antennas. Furthermore, the conductors and dielectrics are represented by inductances, capacitances and resistances, which makes it possible to include additional lumped circuitry into the simulation. The feasibility of using the PEEC method to describe an RFID system has been studied previously in $[13,14]$. It is here expanded to also include the problem of correctly describing the performance of an RFID system as the transponders rotate and move randomly through the reader. This can be simulated effectively using the Monte Carlo method. Using this approach, the number of PEEC simulations is minimised, which reduces the simulation time, whereas at the same time a large number of relative orientations and positions of the reader and transponder may be analysed. The focus in this paper is on RFID systems operating in the $125 \mathrm{kHz}$ band; the results can however also be applied to higher frequencies. The study here is done for two different types of RFID reader antennas and the simulations are compared to the results from measurements on similar systems.

\section{Theory}

The basic principles of an inductively coupled RFID system are shown in Fig. 1. As the transponder comes close to the reader antenna, the voltage ' $V$ out ' will change because of the

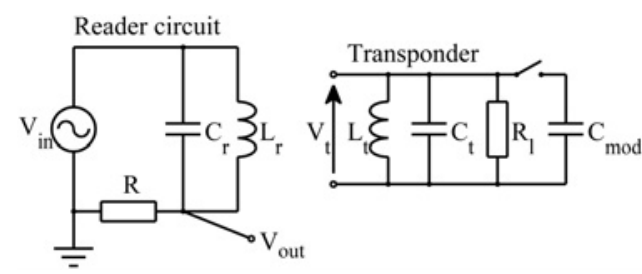

Figure 1 Basic function of an inductively coupled RFID system

The voltage ' $V_{\text {out }}$ ' is the signal used to detect a transponder

mutual inductance between the coils. The simulation approach in this paper is largely based on [13] where this effect was simulated using PEEC for different transponder locations in the vicinity of the reader antenna. It is adapted here to the situation in which a very large number of transponder locations and orientations needs to be simulated and where a complex environment must be taken into account. It is then necessary to minimise the number of electromagnetic simulations by using some suitable approximation in order to reduce the computational load. This is done by approximating the transponder using a magnetic dipole which can be moved around in the interrogation zone of the reader. If the effect of the transponder on the magnetic field created by the reader antenna is small, the analysis of the reader and the transponder can be completely decoupled. Consequently, the voltage induced by the transponder in the reader needs to be small compared to $V_{\text {in }}$ in Fig. 1, which is the case in most passive low-frequency RFID systems. When the properties of the transponder are known, the strength of the signal received by the reader can be calculated using traditional circuit theory. Thus, only one electromagnetic simulation per reader antenna needs to be performed. The approach assumes that the transponder is small compared to the reader antenna and that the voltage induced by the transponder in the reader circuit is small.

\subsection{PEEC method}

This section gives a brief summary of the classical, orthogonal PEEC formulation which is derived from the equation for the total electric field at a point written as

$$
\boldsymbol{E}^{\mathrm{i}}(\boldsymbol{r})=\frac{\boldsymbol{I}(\boldsymbol{r})}{\sigma}+\mathrm{j} \omega \boldsymbol{A}(\boldsymbol{r})+\nabla \phi(r)
$$

where $\boldsymbol{I}$ is the electric current and $\boldsymbol{A}$ and $\phi$ are the electric vector and scalar potentials, respectively. These are given by

$$
\begin{aligned}
& \vec{A}(\boldsymbol{r})=\mu \int_{V^{\prime}} G\left(\boldsymbol{r}, \boldsymbol{r}^{\prime}\right) \boldsymbol{I}\left(\boldsymbol{r}^{\prime}\right) \mathrm{d} v^{\prime} \\
& \phi(\boldsymbol{r})=\frac{1}{\epsilon_{0}} \int_{V^{\prime}} G\left(\boldsymbol{r}, \boldsymbol{r}^{\prime}\right) q\left(\boldsymbol{r}^{\prime}\right) \mathrm{d} \boldsymbol{v}^{\prime}
\end{aligned}
$$

where the Green's function is $G\left(r, r^{\prime}\right)=1 /\left|\boldsymbol{r}-\boldsymbol{r}^{\prime}\right|$. By substituting (2) and (3) into (1), the electric field integral equation at the point $r$ in the conductor is obtained 
according to

$$
\begin{aligned}
\boldsymbol{E}^{\mathrm{i}}(\boldsymbol{r})= & \frac{\boldsymbol{I}(\vec{r})}{\sigma}+\mathrm{j} \omega \mu \int_{V^{\prime}} G\left(\boldsymbol{r}, \boldsymbol{r}^{\prime}\right) \boldsymbol{I}\left(\boldsymbol{r}^{\prime}\right) \mathrm{d} \boldsymbol{v}^{\prime} \\
& +\frac{\nabla}{\epsilon_{0}} \int_{V^{\prime}} G\left(\boldsymbol{r}, \boldsymbol{r}^{\prime}\right) q\left(\boldsymbol{r}^{\prime}\right) \mathrm{d} \boldsymbol{v}^{\prime}
\end{aligned}
$$

Integrating (4) using a suitable inner product, followed by some algebraic manipulations, the Kirchhoff's voltage law for a basic PEEC cell is obtained as

$$
V=R I+L_{p} \mathrm{j} \omega I+P Q
$$

The basic PEEC cell is the building block for more complex PEEC models and one realisation is shown in Fig. $2 b$ of the conducting bar in Fig. $2 a$.

It consists of

- Self-partial inductances between the nodes, shown as $L p_{m m}$ and defined as

$$
L p_{\alpha \beta}=\frac{\mu}{4 \pi} \frac{1}{a_{\alpha} a_{\beta}} \int_{v_{\alpha}} \int_{v_{\beta}} \frac{1}{\left|\boldsymbol{r}_{\alpha}-\boldsymbol{r}_{\beta}\right|} \mathrm{d} v_{\alpha} \mathrm{d} v_{\beta}
$$

for the two volumes $\alpha$ and $\beta$.

- Mutual partial inductances collected in a voltage source $V_{m m}^{L}$ to account for magnetic field couplings to other volumes.

- Node capacitances, $1 / p_{i i}$ and $1 / p_{j j}$, which calculated from the coefficients of potential computed as

$$
p_{i j}=\frac{1}{S_{i} S_{j}} \frac{1}{4 \pi \epsilon_{0}} \int_{S_{i}} \int_{S_{j}} \frac{1}{\left|\boldsymbol{r}_{i}-\boldsymbol{r}_{j}\right|} \mathrm{d} S_{j} \mathrm{~d} S_{i}
$$

for two surfaces $i$ and $j$.

- Mutual capacitances collected in current sources $I_{P}^{i}$ and $I_{p}^{j}$ to account for electric field couplings to other surfaces.
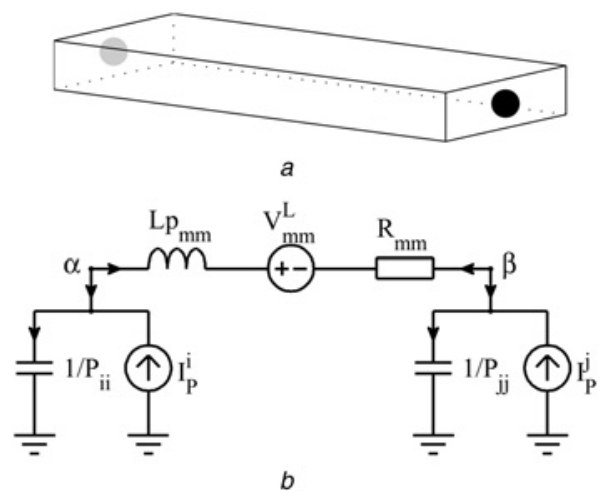

Figure 2 Conducting bar and corresponding PEEC Model

a Conducting bar

$b$ Corresponding PEEC model representation of the conducting bar shown in $a$
- DC resistance defined as

$$
R_{\gamma}=\frac{l_{\gamma}}{a_{\gamma} \sigma_{\gamma}}
$$

In (6) and (8), $a$ is the cross section of the rectangular volume cell normal to the current direction $\gamma$ and $l$ is the length in the current direction. Furthermore, $v$ represents the current volume cells and $S$ the charge surface cells. For a detailed derivation of the method, including the non-orthogonal formulation, see [15].

As seen, the PEEC method is a framework for creating electric equivalent circuit representations of general electromagnetic problems. If a quasistatic approximation is valid, PEECs can be solved to obtain the current- and potential distribution in the studied structure using a free/ commercial simulation program with integrated circuit emphasis (SPICE)-like solver with support of additional electrical components. In this case the magnetic and electric field couplings are translated to mutual partial inductances and mutual capacitances, respectively, since time retardation is neglected, thus, allowing for a mixed circuit/electromagnetic solution suitable for studying RFID systems. However, for the full-wave case when retardation in electromagnetic couplings is of importance, the circuit equations [16] have to be formulated and solved in a specialised solver.

\subsection{Approximation of the transponder}

A simple RFID transponder can be modelled as a current loop and a capacitor. The electric current in the loop can be interpreted as a magnetic dipole with a dipole moment

$$
\boldsymbol{m}=N S I_{t} \hat{\boldsymbol{n}}
$$

where $N$ is the number turns in the loop, $S$ is the area enclosed by the loop, $I_{t}$ is the current in the loop and $\hat{\boldsymbol{n}}$ is the normal to the area enclosed by the loop. If the transponder is small compared to the reader antenna, the magnetic field created by the reader can be assumed to be constant over the whole transponder. It is then possible to calculate the magnetic interaction energy, $U_{\mathrm{rt}}$, using

$$
U_{\mathrm{rt}}=\boldsymbol{m} \cdot \boldsymbol{B}(\boldsymbol{r})
$$

where $\boldsymbol{B}(\boldsymbol{r})$ is the magnetic field at the point $\boldsymbol{r}$ created by the reader antenna [17]. Using the mutual inductance, $M$, between the reader and transponder, the magnetic interaction energy can also be written as

$$
U_{\mathrm{rt}}=M I_{\mathrm{r}} I_{\mathrm{t}}
$$

where $I_{\mathrm{r}}$ is the current in the reader coil. Using (10) and (11) the mutual inductance between the reader and the 
transponder can be written as

$$
M=\frac{N S}{I_{\mathrm{r}}} \hat{\boldsymbol{n}} \cdot \boldsymbol{B}_{r}(\boldsymbol{r})
$$

The current $I_{\mathrm{r}}$ is given by the PEEC-simulation of the system.

By inspection of Fig. 1, the voltage induced by the reader in the transponder is

$$
V_{\text {transponder }}=\frac{\mathrm{j} \omega M I_{\mathrm{r}}}{1+\left(Z_{\mathrm{L}_{\mathrm{t}}} / Z_{\mathrm{t}}\right)}
$$

where $M$ is given in (12), $\omega$ is the angular frequency, $Z_{\mathrm{L}_{\mathrm{t}}}$ is the impedance of the coil in the transponder and $Z_{t}$ is the impedance of the transponder. The impedance $Z_{\mathrm{L}_{\mathrm{t}}}$ of the transponder antenna (denoted as $L_{t}$ in Fig. 1) is typically modelled as an inductance in series with a resistance and the impedance $Z_{t}$ of the transponder is modelled as a capacitance in parallel with a resistance.

The voltage induced by a transponder in the coil of the reader antenna will depend on the current that is induced in the transponder by the reader antenna. This relationship can be written as [18]

$$
V_{\text {ind }}=\mathrm{j} \omega M I_{\mathrm{t}}=-\frac{\omega^{2} M^{2} I_{\mathrm{r}}}{Z_{\mathrm{L}_{\mathrm{t}}}+Z_{\mathrm{t}}}
$$

The transponder communicates with the reader antenna by changing its load, $Z_{\mathrm{t}}$. This is done either by adding an extra resistor in parallel with the circuit (ohmic load modulator) or by adding a capacitance in place of the resistor (capacitive load modulator). This is shown in Fig. 1 for a capacitive load modulator. In both of these cases both the amplitude and the phase of the voltage, $V_{\text {ind }}$, induced in the reader coil is changed. To determine the readability of the transponder, it is therefore necessary to calculate the difference in the induced voltage $V_{\text {ind }}$ with the modulator on or off. The signal strength can thus be written as

$$
V_{\text {ind }}=-\omega^{2} M^{2} I_{\mathrm{r}}\left[\frac{1}{Z_{\mathrm{L}_{\mathrm{t}}}+Z_{\mathrm{t}, 1}}-\frac{1}{Z_{\mathrm{L}_{\mathrm{t}}}+Z_{\mathrm{t}, 2}}\right]
$$

where $Z_{\mathrm{t}, 1}$ and $Z_{\mathrm{t}, 2}$ are the transponder impedances when the load modulator is connected and unconnected, respectively.

\subsection{System simulation}

The parameter of interest when designing an RFID system is the detection rate of RFID transponders. In order for a transponder to be detected by the reader circuit, two criteria must be fulfilled. Firstly, the magnetic field at the location of the transponder must be sufficiently strong to meet the transponder's power needs. Secondly, the voltage induced by the transponder in the reader circuit must be high enough during a long enough time to be detected over the noise. Thus, in order for a successful detection of an RFID transponder the voltages in (13) and (14) must be above some threshold value for a certain period of time.

As can be seen above, the voltage induced by an RFID transponder in a reader depends on both the magnetic field at the location of the transponder and the orientation of the transponder with respect to the field. By using the magnetic dipole approximation only one simulation of the reader antenna and circuitry is needed in which the magnetic field in the vicinity of the reader antenna is calculated. The read rate of RFID transponders can then be estimated using a Monte Carlo simulation.

The Monte Carlo method is suitable when the system to be simulated consists of a large number of particles whose behaviour (location, speed, rotation, etc.) can be described using statistical mechanics [19]. In the case of an RFID system, this can be applied by assuming that each RFID transponder is a magnetic dipole as described above. At a given time, $t$, the transponder will be located at a point, $r$, and oriented in the direction $\hat{\boldsymbol{n}}$. A transponder's starting position and orientation at time $t_{0}$ and the subsequent positions and orientations at time $t_{n}$ is determined using knowledge about the statistical distribution of the transponders' position, velocity and orientation.

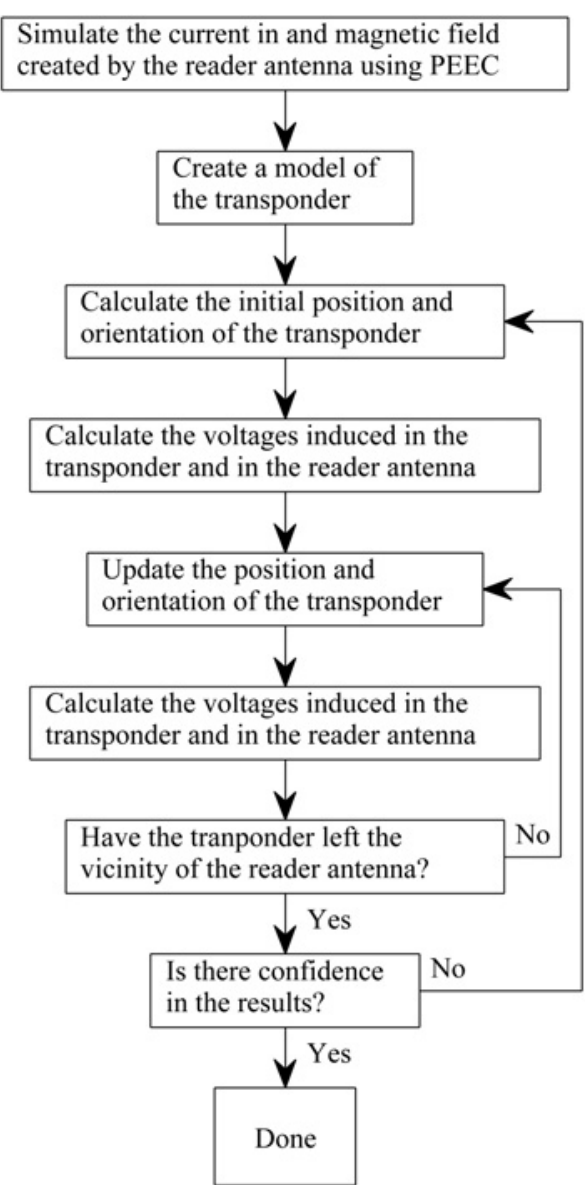

Figure 3 Flowchart of the simulation procedure 
The simulation of a complete RFID system using the proposed method is outlined in Fig. 3. In the first step the performance of the reader antenna and circuit is simulated using PEEC. In the second step the transponder is modelled as described in Section 2.2. A random starting position and orientation of the transponder is then chosen from an appropriate distribution and the induced voltages in the transponder and reader antenna are calculated using (13) and (14), respectively. This is repeated for updated positions of the transponder until it has left the vicinity of the reader antenna. The procedure is repeated for new starting positions and orientations until there is sufficient confidence in the results.

The procedure outlined above is general and requires detailed information both about the reader and transponder circuitry and the construction of the transponder antenna. If this information is not available, the procedure can be simplified by only analysing some parameter of interest (e.g. the maximum voltage induced by each transponder in the reader circuit). The method could then still be a powerful means of comparing different antenna geometries even if the knowledge about the system is limited. This is the approach used in the later sections of this paper.

\section{Simulations}

The method presented above was used to simulate and compare the performance of two different antenna configurations for a system operating in an industrial environment. In this system, the transponders are placed among iron ore pellets on a conveyor. The purpose of the analysis is to compare two different antenna configurations to determine which is most likely to detect most transponders. As indicated earlier, the focus is on performing a qualitative analysis of the differences between the antenna geometries rather than describing the properties of each antenna in absolute numbers.

The two antenna configurations are shown in Fig. 4. The transponders are assumed to move on a straight line through the grey region in the middle of the figures (in random position in this region). Also, it is assumed that the transponders are oriented in an arbitrary direction without rotating. In the first design shown in Fig. $4 a$, denoted antenna 1 , the reader antenna is mounted around the conveyor and is shaped in order to obtain as close as possible to the pellets where the transponders are located. This antenna is compared to the second design shown in Fig. $4 b$, denoted antenna 2 , in which the entire reader antenna is located under the conveyor. The antenna is bent slightly as a saddle in order to move the conductors closer to the pellets.

In the measurement presented in the next section the transponders used were $22 \times 4$ and $12 \times 2.12 \mathrm{~mm}$. The actual coil in the transponder is slightly smaller than these dimensions. The transponders are assumed to be matched to

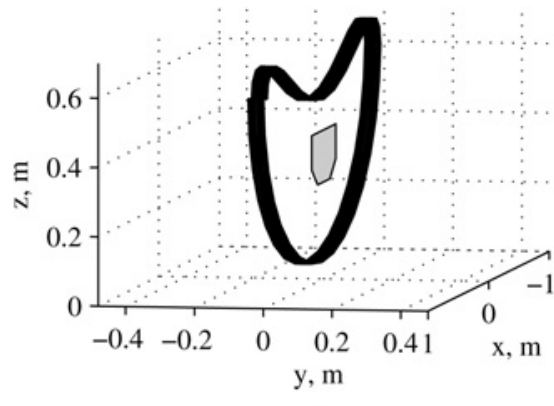

a

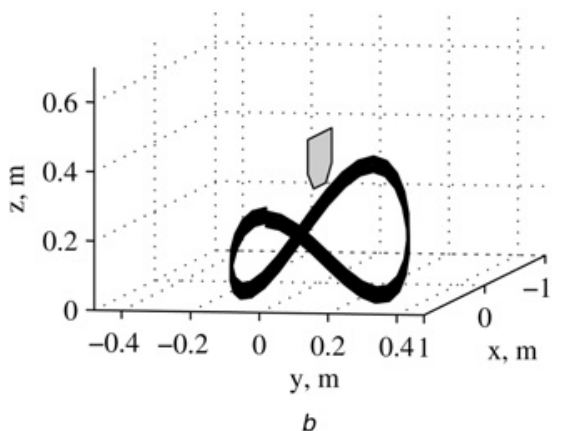

Figure 4 Two different RFID reader antenna configurations

a Reader antenna is mounted around the conveyor as a loop $b$ Reader antenna is mounted below the conveyor as a saddle The transponders move on a straight line through the gray region

$125 \mathrm{kHz}$ and modulated using a capacitive load of equal size as the one used for the matching. In the simulation, 300 loops of coil with a diameter of $4 \mathrm{~mm}$ were used and the inductance of the coil is then approximately $1.1 \mathrm{mH}$. It is assumed that this coil has an internal resistance of $5 \Omega$. Further, the load of the transponder is assumed to be $5 \mathrm{k} \Omega$. This choice does, however, not affect the final results since all induced voltages are normalised to the maximum obtained value.

For the PEEC-simulation, only the centre frequency, $125 \mathrm{kHz}$, of the band was considered although there is in reality a bandwidth of about $6 \mathrm{kHz}$. Also, the relatively simple reader circuit shown in Fig. 1 was used. This is not the same as the one used in the measurements where the circuitry is significantly more complex. These approximations are motivated by the fact that the property of interest is the shape and orientation of the reader antennas.

Fig. 5 shows how the voltage induced by the transponder in the reader antenna changes as the transponder moves through the read area. The transponder is assumed here to move on a straight line in the upper part of the region shown in Fig. 4. The voltage is shown for both antennas for the case when the transponder is oriented perpendicular to the plane spanned by antenna 1 ( $x z$-plane in Fig. 4 ) and when it is oriented perpendicular to the the plane spanned by antenna 2 ( $x y$-plane in Fig. 4). It can be seen that the induced voltage is strongest when the transponder is oriented perpendicular to the reader antenna which is expected since they are in this case most likely oriented along the magnetic field lines. In the case when the 


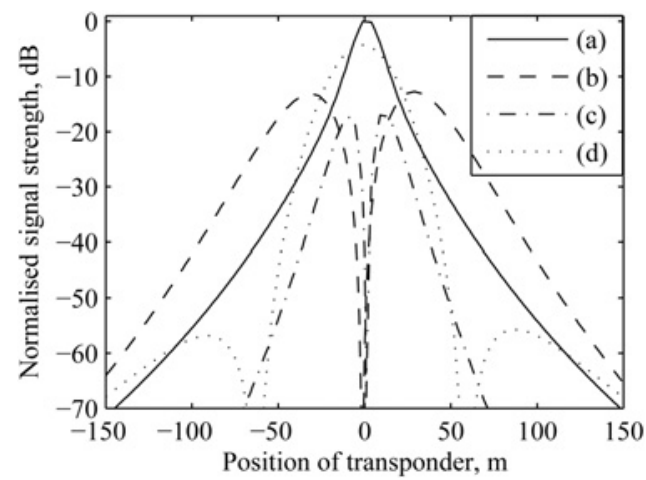

Figure 5 Induced voltage for different location of the transponder in Fig. 4

$a$ and $b$ Antenna mounted around the conveyor with the transponder oriented perpendicular to- and in the plane of the antenna, respectively. Results $c$ and $d$ Antenna mounted below the conveyor with the transponder oriented in- and perpendicular to the plane of the antenna, respectively

transponder is oriented in the same plane as the reader antennas the induced voltage is significantly lower.

The full Monte Carlo simulation was done for 20000 transponders with different orientation and position of the transponders as described above. It is assumed here that the speed of the transponders is low so that there is enough time for the reader circuit to detect them. The parameter of interest in this case is the maximum induced voltage for each transponder. This is shown in Fig. 6 for the reader antenna

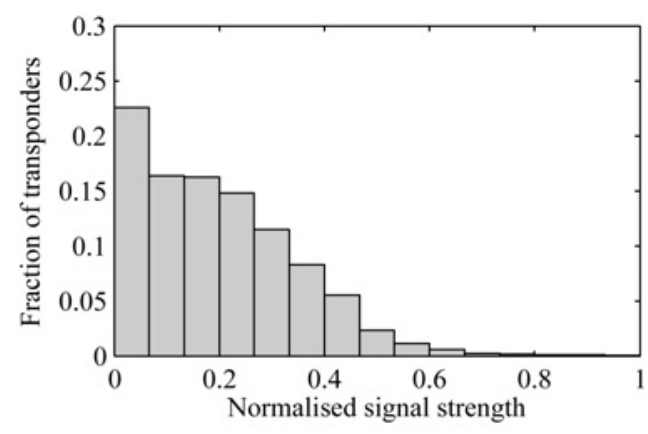

a

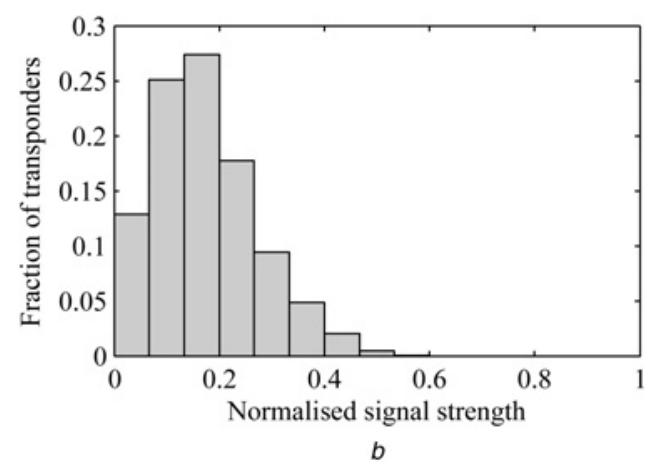

Figure 6 Histogram of the normalised maximum induced voltage found by Monte Carlo simulation

$a$ When the antenna is mounted around the conveyor

$b$ When the antenna is below the conveyor mounted around the conveyor (a) and for the reader mounted below the conveyor (b). The induced voltage is here normalised to the maximum detected value since the main interest is in comparing the two antenna configurations. This also simplifies the comparison between the simulations and the measurements presented in the next section.

The spread of the maximum signal strength is clearly larger when antenna 1 is used compared to antenna 2 . This is due to the fact that this antenna gives a strong, evenly distributed field in the region where the transponders are located. Furthermore, the direction of the field does not change significantly which means that the orientation of the transponders greatly affects the induced voltage. Since the transponders are oriented randomly a large spread is expected for this antenna geometry. Antenna 2 will produce greater variations in the direction of the field along the path of the transponder and the orientation of these will therefore be somewhat less important to the received signal strength. This is the reason for the more collected distribution. It can also be noted that the maximum detected signal strength is lower for antenna 2 than for antenna 1 . This is due to the slightly larger distances between the transponder and the antenna.

\section{Measurements}

The measurements were performed on 2 June 2009 at the LKAB facility in Kiruna, Sweden using the similar antenna systems and transponders as described in the previous section. During the measurements a total of 124 large transponders $(22 \mathrm{~mm})$ and 150 small $(12 \mathrm{~mm})$ were dropped among the raw material (iron ore pellets) on a conveyor. The speed of the conveyor is about $3 \mathrm{~m} / \mathrm{s}$. The two antennas were located at a relatively large distance from each other and the coupling between them is therefore negligible. Once on the conveyor, the transponders do not rotate and thus it can be assumed that the orientation of the transponders is the same in both measurements.

Of the $22 \mathrm{~mm}$ transponders dropped on the conveyor about $61 \%$ were detected by antenna 1 and $77 \%$ by antenna 2. The distribution of the maximum signal strengths does however differ considerably between the two antenna configurations, which can be seen in Fig. 7. This was also verified using a one-way analysis of variance (ANOVA) test [20] which showed that there is a significant difference between the mean values of the maximum detected signal strengths between the two antennas.

The difference between the distributions is similar to what was seen in the simulations, with antenna 1 having a more dispersed distribution than antenna 2. Obviously, only the detected transponders reported any maximum signal strength and therefore no very weak transponders are seen in the measurement results. Also, a transponder that gives a signal which is above the threshold of the reader circuit may still be missed if it is in the vicinity of the reader 


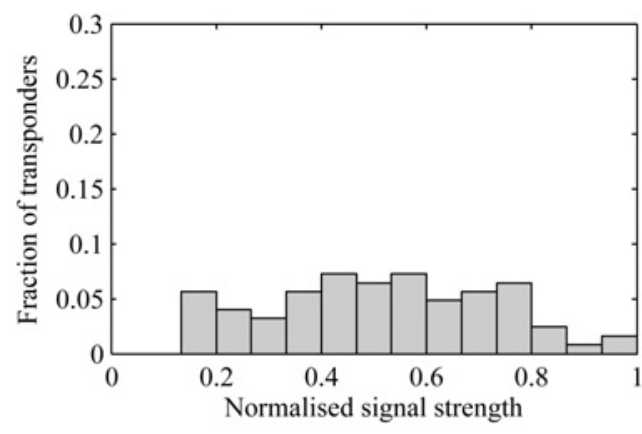

a

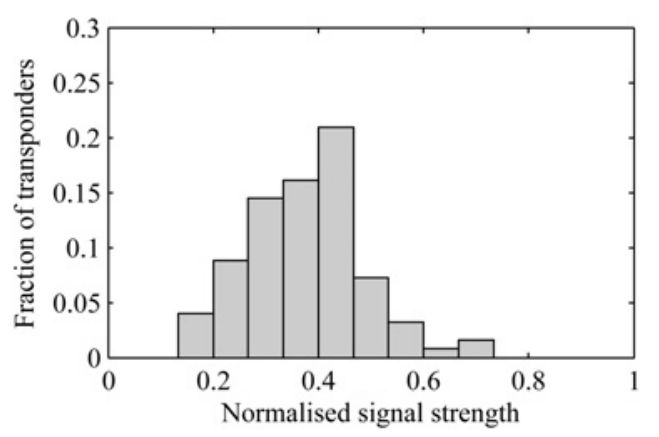

$b$

Figure 7 Histogram of the normalised maximum induced voltage found by measurements using $22 \mathrm{~mm}$ transponders

$a$ When the antenna is mounted around the conveyor $b$ When the antenna is below the conveyor

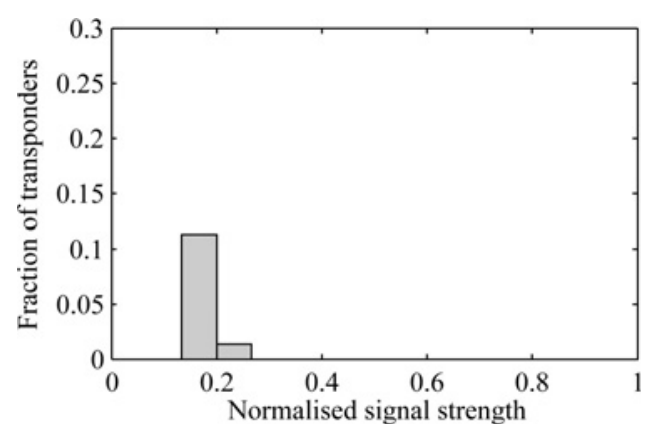

Figure 8 Histogram of the normalised maximum induced voltage found by measurements using $12 \mathrm{~mm}$ transponders

antenna for too short a time. Therefore fewer of the weak transponders will be detected, which will result in the peak being shifted towards higher values.

Of the $12 \mathrm{~mm}$ transponders, antenna 1 detected only $13 \%$ of the transponders which is shown in Fig. 8, whereas antenna 2 did not detect any transponders. The main reason for this is that the coil in the $12 \mathrm{~mm}$ transponder is smaller and therefore interacts more weakly with the reader antennas. For both these measurements the same type of distribution as seen for the $22 \mathrm{~mm}$ transponders could be expected. The results are also consistent with the results seen in both simulations and measurements for the $22 \mathrm{~mm}$ transponders, with the threshold of the circuit being above the maximum signal strength seen for antenna 2 , in which case very few transponders would be detected in antenna 1. If, on the other hand, the size of the transponders would be increased, it could be expected from the simulation results that antenna 2 performs better in terms of number of detected transponders since the threshold may then be below the peak seen in the distribution in Fig. $6 b$.

\section{Conclusions}

A method based on the PEEC and the Monte Carlo methods which can be used to describe the performance of complex RFID systems with moving and rotating transponders has been presented. The method has been used to compare two different antenna configurations and it has been shown that the results are similar to the ones obtained using measurements in an industrial environment. Owing to the magnetic dipole approximation used, the method is best suited for situations where the dimensions of the transponders are much smaller than the reader antenna.

In the present application it is advantageous to use the PEEC method to obtain the magnetic field of the reader antenna since the integral formulation makes it efficient in situations when the structures contain a lot of air. Also, the ability to include circuits in the simulation is convenient when simulating passive RFID systems. In other applications a similar approach could be adopted using other methods than PEEC. This is something that should be studied further.

Being able to describe the behaviour of a RFID system in statistical terms is advantageous since it gives a means of predicting the read rate of a given antenna. To obtain the signal strength in absolute numbers would however require detailed information of all parts of the system, including transponder, reader circuits, noise levels and so on. Even though this information may not be available the simulation method is still a powerful tool that qualitatively can describe the different properties of the available antenna configurations.

\section{References}

[1] KVARnström B., Vanhatalo e.: 'Using RFID to improve traceability in process industry', J. Manuf. Technol. Manage., 2010, 21, (1), pp. 139-154

[2] ClaRke R.H., TWEDE D., TAZelAAR J.R., BOYeR K.K.: 'Radio frequency identification (RFID) performance: the effect of tag orientation and package contents', Packag. Technol. Sci., 2006, 19, pp. 45-54

[3] MITSUGI J., HADA H.: 'Experimental study on UHF passive RFID readability degradation'. Proc. Int. Symp. on Applications and the Internet Workshops, Phoenix, Arizona, USA, January 2006

[4] RAhMATI A., ZHONG L., hiltunen M., JANA R.: 'Reliability Techniques for RFID-based object tracking applications'. 
Proc. IEEE/IFIP Ann. Int. Conf. on Dependable Systems and Networks, Edinburgh, UK, 2007

[5] MO L., ZHANG H.: 'RFID antenna near the surface of metal'. IEEE Int. Symp. on Microwave, Antenna, Propagation, and EMC Technologies for Wireless Communications, Hangzhou, China, August 2007

[6] MUehlmann U., MANZI G., WIEDNig G., BuChmann M.: 'Modeling and performance characterization of UHF RFID portal applications', IEEE Trans. Microw. Theory Tech., 2009, 57, (7), pp. 1700-1706

[7] KaWdungta S., PHONGChaRoenPanich C., tORRUNGRUENG D.: 'Novel design of double loop antennas by using a shifted gate for the LF-RFID system'. Int. Symp. on Antennas and Propagation, Taipei, Taiwan, October 2008

[8] LEONG K.S., NG M.L., COLE P.H.: 'Operational considerations in simulation and deployment of RFID systems'. Proc. IEEE Int. Zurich Symp. on Electromagnetic Compatibility, Zurich, Switzerland, 2006

[9] OLSSON T., KOPTIOUG A.: 'Statistical analysis of antenna robustness', IEEE Trans. Antennas Propagat., 2005, 53, (1), pp. $566-568$

[10] RUEHLI A.E.: 'Inductance calculations in a complex integrated circuit environment', IBM J. Res. Dev., 1972, 16, (5), pp. $470-481$

[11] RUEHLI A.E., BRENNAN P.A.: 'Efficient capacitance calculations for three-dimensional multiconductor systems', IEEE Trans. Microw. Theory Tech., 1973, 21, (2), pp. 76-82
[12] RUEHLI A.E.: 'Equivalent circuit models for three dimensional multiconductor systems', IEEE Trans. Microw. Theory Tech., 1974, 22, (3), pp. 216-221

[13] LINDGREN T., EKMAN J.: 'Design and evaluation of RFID systems using the partial element equivalent circuit method'. Int. Symp. on Antennas and Propagation, Taipei, Taiwan, October 2008

[14] REINHOLD C., SCHOLZ P., JOHN W., HILlERINGMANN U.: 'Efficient antenna design of inductive coupled RFID-systems with high power demand', J. Commun., 2007, 2, (6), pp. 14-23

[15] ANTONINI G., RUEHLI A.E.: 'Non-orthogonal PEEC formulation for time and frequency domain EM and circuit modeling', IEEE Trans. EMC, 2003, 45, (2), pp. $167-176$

[16] ANTONINI G., EKMAN J., ORLANDI A.: 'Full wave time domain PEEC formulation using a modified nodal analysis approach'. Proc. EMC, Europe, The Netherlands, 2004

[17] WANGSNESS R.: 'Electromagnetic fields' (John Wiley \& sons, 1986, 2nd edn.)

[18] FINKENZELLER K.: 'RFID handbook' (Wiley, England, 2003, 2nd edn.)

[19] GARCIA A.L.: 'Numerical methods for physics' (Prentice Hall, 1999, 2nd edn.)

[20] MONTGOMERY D.C.: 'Design and analysis of experiments' (John Wiley \& sons, 2005, 6th edn.) 